\title{
Analysis on Clinical Features and Esophageal Motility in Elderly Patients with Gastro-Esophageal Reflux Disease
}

\author{
Mengdie Shen, Shanshan Chen, Lina Meng* \\ The First Affiliated Hospital of Zhejiang Chinese Medical University, Hangzhou, 310006, China \\ * Corresponding Author: Lina Meng
}

Keywords: Esophageal motility, Gastro-esophageal reflux disease, Elderly patients

\begin{abstract}
To study the relationship between acid reflux and esophageal motility in patients with gastroesophageal reflux disease. For patients who met the diagnostic criteria of GERD, the author divided them into two groups: the physiological acid reflux group and the pathological acid reflux group. The esophageal motility was compared between the two groups based on the results of $\mathrm{pH}$ monitoring of $24 \mathrm{~h}$ esophagus. According to the results, the difference between the two groups was statistically significant $(\mathrm{P}<0.05)$. There was no significant difference in the speed of esophageal peristalsis wave and the proximal and middle esophageal body pressure between the two groups. Therefore, pathological acid reflux in GERD patients is closely related to LESP, but not related to esophageal peristalsis wave propulsion speed, proximal and middle esophageal body pressure.
\end{abstract}

\section{Introduction}

The cause of GERD is multifactorial, which includes defects in reflux barriers, abnormal esophageal clearance, changes in esophageal mucosal resistance, and delayed gastric emptying [1]. Among them, mucous damage is mainly caused by gastric contents (gastric acid, pepsin) and even duodenal fluid reflux. The movement of the esophagus and stomach is mainly through the contraction of the nerve and muscle to complete the peristalsis and conduction. Under physiological condition, the intragastric pressure is higher than the intraesophageally pressure in the thoracic cavity. The lower esophageal sphincter shrinks in resting state. Maintaining a certain pressure is an important barrier to anti reflux. It plays a role in preventing gastrointestinal contents from reflux. Along with the growth of age, esophageal smooth muscle correspondingly changes in structure. LES relaxation and lower esophageal sphincter resting pressure (LESP) decrease. Therefore, gastroesophageal reflux is easy to happen. On the other hand, the normal pressure and anterograde of esophageal body is the main force to clear away reflux in time. In the elderly, esophageal motor dysfunction and esophageal body clearance were significantly weakened, which could not clear the reflux in time, resulting in the prolonged contact time between reflux and reflux, which aggravated the damage of reflux to esophageal mucosa. The decrease of saliva and other secretions in elderly people leads to a decrease in acid clearance, and a decrease in the proliferation and repair ability of esophageal epithelium, which leads to a higher incidence of GERD in the elderly. At the same time, the elderly suffering from various diseases, often oral medications, such as theophylline, anticholinergic, antidepressants, sedative drugs, calcium antagonists, prostaglandins and other drugs, can directly damage the esophageal mucosa or LES reduce the pressure caused increased acid exposure, obviously promote gastroesophageal reflux [2].

\section{Objects and Methods}

\subsection{Objects}

In this group, there were 72 cases of 48 males and 24 females, 56 cases aged 60 69 years, 12 cases of 70 79 years, 4 cases over 80 years old, and $17 \mathrm{~d} \sim 16$ years. Heartburn, reflux for typical symptoms; only without chest pain, heartburn, reflux for atypical symptoms; for asthma, sore throat, cough and 
other special manifestations, easily misdiagnosed as angina, asthma, pharyngitis, misdiagnosis duration is $7 \mathrm{~d}$, the longest 9 years. The coexistence of angina and bronchial asthma is easy to leak.

\subsection{Methods}

Retrosternal or epigastric burning sensation, chest pain, belching and other symptoms, or refractory asthma, chest pain, cough, sore throat, etc. There are signs of gastroesophageal reflux by endocytosis and gastroscopy. It is effective by using acid suppressant and gastric motility medicine. Raise the head side of the bed $20 \sim 30 \mathrm{~cm}$ during sleep. First with ranitidine $150 \mathrm{mg}, 2$ /d, $300 \mathrm{mg}$ or 1 times every night, with the application of prokinetic drugs, domperidone $10 \mathrm{mg}$ or propulsive $5 \sim 10$ $\mathrm{mg}, 3$ /d, for 8 weeks. If the effect is not good, ranitidine can be changed to omeprazole $40 \mathrm{mg}, 2$ times /d, 4 weeks for a course of treatment.

Often associated with postural changes of heartburn, nausea, chest pain, and dysphagia or swallowing pain, such as patients in the supine or bending when the emergence of heartburn and sit up or swallow liquid can alleviate the diagnosis can be made. The evidence for esophageal $\mathrm{pH}$ monitoring, endoscopic and imaging, and esophageal injury can be diagnosed. For those with atypical reflux symptoms, a comprehensive analysis of endoscopy, esophageal dynamics and experimental treatment should be combined to make a diagnosis. Gastroesophageal reflux disease is a recurrent chronic disease. Some patients need long-term treatment to prevent the occurrence and recurrence of complications. Therefore, maintenance treatment is necessary. Maintenance treatment can be divided into continuous treatment, intermittent treatment and on-demand treatment. Gastroesophageal reflux disease is often used to promote gastric motility and anti-acid drugs in clinical treatment. In principle, it is based on the complete relief of clinical symptoms, combined with the condition, and selects drugs with high efficiency and small adverse reactions, and gives relatively long reduction therapy. Studies have shown that small doses of proton pump inhibitors are more effective in preventing relapse than other drugs.

For patients with mild symptoms and less frequent relapse after withdrawal, before the autonomic function test, $12 \mathrm{~h}$ banned drinking coffee, tea and alcohol, banned smoking, and banned any drugs that affect blood pressure and heart rate, ensuring adequate sleep and avoiding strenuous exercise. The test was carried out in a comfortable and quiet environment. The case group and the control group respectively, heart rate variability, the Beijing science and technology flourier autonomic detection system, take the method of analysis of $5 \mathrm{~min}$ short range frequency domain, frequency domain parameters of low frequency power and high frequency power. The ratio of high frequency, low frequency power and low, high frequency power is recorded to reflect the tension ratio of sympathetic and vague nerve, and the rate of heart rate variability in short range frequency domain is analyzed.

\section{Results}

\subsection{Clinical Features}

GERD is a common disease of digestive system, mainly for upper abdominal pain, acid regurgitation, chest pain, and endoscopic examination of esophageal mucosa damage. The elderly is a high incidence of this disease. Not only due to the organic reason of body, in recent years, it is considered that mental and psychological factors may play an important role in the pathogenesis. Studies have confirmed that psychosocial factors induce the sensation of esophagus and visceral Gao Min through the peptide axis of brain gut, resulting in slight irritation in the esophagus, which can induce fever, pain and other symptoms on the upper abdomen. Psychological and behavioral factors are important factors that affect the severity of the patients' symptoms and the frequency of their visits. Research shows that psychological factors play an important role in the perception of symptoms, including anxiety, depression, somatization there are significant correlations, in elderly patients with depression and anxiety, the somatic symptoms of GERD susceptibility than patients with good mood. Psychosocial factors can aggravate GERD symptoms, and bad mood can affect the physiological function of the esophagus, and the patient's response to the routine treatment of acid suppressor is 
poor.

Neuroendocrine regulation of digestive system, high nerve center of the autonomic nervous system by sympathetic parasympathetic nerve, regulating the normal activities of the system, under normal conditions, sympathetic nerve and vagus nerve in a dynamic balance, can cope with various physiological needs of the body. Under the condition of stress or emotional changes, the autonomic nervous system's activity rule is disturbed, and the functions of its nervous system are disturbed, leading to the corresponding symptoms. Abnormal mental and psychological factors and autonomic nervous dysfunction may play a role in the pathogenesis of functional digestive diseases. Whether mental and psychological factors are abnormal through autonomic nervous dysfunction to make psychosocial factors somatization still need further study. The general elderly can achieve the purpose of treatment by conservative treatment in internal medicine. Anti-reflux surgery can be used for the patients who have not been treated by internal medicine. Unfortunately, many surgical patients still need acid treatment to achieve the best symptom relief. Therefore, the surgical treatment of GERD is still cautious [3].

The elderly GERD patients with long-term pain and tension, anxiety, and anxiety, and even feel hopeless, then you need to patiently and carefully to the elderly explains the causes of stomach discomfort, reduce the church methods and skills of stomach discomfort, alleviate the fear. Consult with family and participate in collective activities, such as meeting friends, to increase the sense of belonging of the old.

The meal to help the elderly take a high position, given sufficient time, and told the old man eating slower, attention should be focused on, every time a small amount of food, and the swallow and then to another port. A small number of meals should be replaced by many three meals. Food processing should be soft rotten, by boiling, stewing, boiling, steaming and cooking methods, food can be processed into a paste or minced meat and vegetable puree mud. In addition, according to the individual's eating habits, food color, flavor and taste, shape sensory traits, to stimulate the appetite, food collocation should be diversified, the main non-staple food is reasonable, considering the thickness. At the same time, pay attention to avoid eating overfull, and try to reduce the intake of fat. High acid food can damage the esophageal mucosa, and the citrus juice and other acidic foods should be restricted. Stimulant food can cause an increase in gastric acid secretion, and should reduce the intake of alcohol, tea, coffee and other foods. More severe chest pain patients with angina pectoris, GERD is difficult to define, or both, the differential points are: GERD angina occurred in the postprandial $1 \mathrm{H}$, duration $4 \sim 5 \mathrm{~min}$; sleep supine, chest pain worsened, and sat up and stand up pectoris eased most reflux signs; gastroscopy; antacids and prokinetic drugs effective; the original suffering from angina should be considered according to the GERD treatment [4].

\subsection{Esophageal Motility}

GERD was associated with many factors, including the lower esophageal sphincter pressure, low frequent transient lower esophageal sphincter relaxation, esophageal acid clearance time prolonged. The lower esophageal sphincter function as the main anti reflux barrier, can effectively prevent the gastric contents into the esophagus. LES low pressure can lead to stomach or duodenal contents into the esophagus, and esophageal mucosal damage caused by a series of symptoms. Studies have shown that LES low tension may be an important factor for gastroesophageal reflux cannot be ignored. The elderly due to age effects of physiological function decreased gradually due to the pressure of LES decreased, anti-reflux barrier dysfunction. The results showed that the elderly and young two disease group LESP decreased, and the elderly group LESP was significantly lower than that in the young group ( $\mathrm{P},<0.001$ ) in elderly RE group was the most significant, consistent with the reported literature. And the old NERD group and elderly group RE, the difference between the two groups was significant $(\mathrm{P},<0.05)$ that is important in the pathogenesis of GERD LESP decreased, the elderly due to age, LES anti reflux barrier dysfunction, leading to increased esophageal acid exposure, esophageal mucosal damage aggravated with esophagitis LES dysfunction is more obvious. We speculate that the elderly than middle-aged people are more likely to suffer from gastroesophageal reflux disease. 
The effective way of esophageal body peristalsis and contraction of normal esophageal clearance is conductive function of normal protection. When the esophageal motility function disorder is not timely and effective will back up into the esophagus stomach and duodenal contents removed, so that the esophageal mucosa and bile acid exposure time prolonged on esophageal mucosa injury. The severity of esophageal mucosa injury and esophageal motility function abnormality rate was positively correlated with the incidence of severe reflux esophagitis and esophageal body movement the abnormal patients were significantly higher than that of NERD, but research shows that no causal relationship between esophageal mucosal injury and the degree of esophageal body motility disorder. Our study shows that the elderly Group LESP was significantly lower than that of the middle age group, the esophageal body motility parameters of peristaltic wave velocity, duration and two indexes of middle-aged people was significantly different $(\mathrm{P}<0.05, \mathrm{P}<0.01)$, suggesting that esophageal motility function in the elderly is weakened, resulting in acid clearance ability were decreased. The results of this study also showed that young two disease groups esophageal PD were shorter than two senile disease in middle-aged group, NERD group, compared with the old RE group, was statistically significant $(\mathrm{P}<0.05)$ suggest that the elderly esophageal motility dysfunction and the severity of gastroesophageal reflux was closely correlated.

Excessive esophageal mucosal acid exposure is another important factor in the pathogenesis of GERD, and the 24hpH monitoring can accurately record esophageal acid reflux. Studies have reported with abnormal acid esophagitis in patients with GERD than those without exposure to severe esophagitis. Esophageal mucosal damage easily by acid reflux serious now and the esophageal motility disorder in GERD patients. Age as an independent risk factor in the pathogenesis of GERD on esophageal acid reflux also has certain influence. Compared with other groups. The elderly, many indexes of esophageal acid exposure increased significantly. The research results show that in the two indexes of supine $\mathrm{pH}<4$ score and the percentage of time elderly disease group were significantly higher than that of the middle-aged group $(\mathrm{P}<0.05)$ was not statistically significant. Other indicators suggest the presence of significant gastroesophageal reflux in elderly patients with GERD esophageal acid exposure to another people Group. But we found that despite this, elderly GERD patients with typical symptoms of gastroesophageal reflux is not obvious in middle-aged people, and endoscopic esophageal mucosal damage is often serious than the latter, extraesophageal symptoms more, suggesting that we should pay more attention to the clinical diagnosis of atypical GERD features in elderly patients.

\section{Conclusions}

The occurrence of pathological acid reflux in GERD patients is closely related to the decrease of LESP and esophageal motility disorders. The effect of esophageal peristalsis wave speed on esophageal acid clearance remains to be further explored. The elderly GERD is one of the most common and frequently occurring diseases of the digestive system, which seriously perplex the life and health of the patients. After the understanding and learning of the elderly GERD, we hope to cause great attention and reduce the incidence of GERD in the elderly.

\section{References}

[1] Niu Xiaoyu, Zhang Mei, She Jing. Influence of Different Esophageal Motor Abnormalities on Gastro-Esophageal Reflux Disease [J]. Journal of Nanchang University Medical Sciences, 2015, 55(4): 33-39.

[2] Li Ping, Qiu Zhongwei. Lnvestigation of Common Esophageal Motility Disorders in Patients With Refractory Gastroesophageal Reflux [J]. Guangdong Trace Elements Science, 2016, 23(5): 27-30.

[3] Hou Yanhong, Zhang Lin, Zhang Kunpeng, et al. Psychological alterations and autonomic nervous dysfunction in elderly patients with gastro-esophageal reflux disease [J]. Chinese Journal of 
Health Care and Medicine, 2012, 14(6): 433-435.

[4] Shi Lei, Wang Wei. Diagnosis and treatment of gastroesophageal reflux disease in the elderly [J]. Chinese Journal of Diagnostics (Electronic Edition), 2017, 5(2): 73-79. 